\title{
ORNAMENTACION ESPORAL (MEB) Y TAXONOMIA DE ALGUNAS ESPECIES DE Cortinarius (AGARICALES, BASIDIOMYCETES) EN BOSQUES NATIVOS DE LA $X$ REGION - CHILE
}

\author{
(Sporal ornamentation (MEB) and taxonomy of some strains of Cortinarius \\ (Agaricales, Basidiomycetes) in native forests of X Region-Chile)
}

\author{
E. Valenzucla* \& G. Moreno ** \\ * Instituto de Microbiología, Facultad de Ciencias, \\ Universidad Austral de Chile, Casilla 167, Valdivia, Chile. \\ ** Departamento de Biologia Vegetal (Botánica), \\ Facultad de Ciencias, Universidad de Alcalá, \\ Alcalá de Henares 28871, Madrid, España.
}

Palabras clave: Cortinarius, Nothofugus, taxonomía, ecología, Chile. Key words: Cortinarius, Nothofagus, ta.sonomy, ecology, Chile.

\section{RESUMEN}

Se citan y comentan 15 especies del género Cortinarius recogidas en bosques de Nothofagus de la $\mathrm{J}$ Region de Chile. Se aportan microfotografias al microscopio electrónico de barrido (MEB) de la ornamentación esporal y datos sobre la ecologia y corologia de las especies citadas. Se citan por primera vez para Chile Cortinarius fuscus Moser y Cortinarius pugionipes Moser. Se proponen siete nuevas combinaciones: Cortinarius amoenus ( $M$.oser \& Horak) Valenzuela \& $G$. Moreno, Cortinarius flavofucatus (M foser \& Horak) Valenzuela \& G. Moreno, Cortinarius icterinus (Horak) G. Moreno \& Valenzuela, Cortinurius nahuelbutensis (Garrido)Valenzuela \& G. Moreno, Cortinurius oliveoicterinus (Moser)Valenzuela \& G. A Ioreno, Cortinarius semipellucidus (M Toser) G. Moreno \& Valenzuela y Cortinarius teresse (Garrido) G. Moreno \& Valenzuela.

\section{INTRODUCCION}

En Chile las especies del género Cortinarius (Pcrs.) Gray son los micorrizógenos más abundantes de las distintas especies arbóreas de Nothofagus. Los" cortinarios" son muy diversos en la zona centro-sur del país, área de distribución natural de las diferentes especies de Nothofagus del hemisferio sur y del presente estudio. De acuerdo a Garrido (1985) han sido citadas para Chile 96

\section{SUMMARY}

A total of 15 species of genus Cortinarius collected in forests of Nothofagus from the X Region of Chile are mentioned and commented. Photomicrographs of the sporal ornamentation taken by microscopy SEM are given. Cortinarius fuscus Moser and Cortinarius pugionipes Moser are recorded for the first time in Chile. Notes on their ecology and chorology are also added. Seven new combinations are proposed: Cortinarius amoenus (Moser \& Horak) Valenzuela \& G. Moreno, Cortinurius flavofucutus (M. Foser \& Horak) Valenzuela \& G. Moreno, Cortinarius icterinus (Horak) G. Moreno \& Valenzuela, Cortinarius nahuelbutensis (Garrido) Valenzuela \& G. Moreno, Cortinarius oliveoicterinus (Moser) Valenzuela \& G. Moreno, Cortinarius semipellucidus (Moser) G. Moreno \& Valenzuela and Cortinarius teresse (Garrido) G. Moreno \& Valenzuela.

especies de Cortinurius, la gran mayoría fueron descritas por Moser \& Horak (1975) y Horak (1980), desde los bosques de Nothofagus andinopatagonicos argentinochilenos.

En estudios posteriores Garrido (1988), cita 15 nuevas especies de Cortinarius, que fructifican en los bosques de Nothofagus comprendidos entre la VII y IX Región de Chile, además este autor cita 9 especies por primera ve $\angle$ para Chile. 
Valenzuela el il (1992, 1994), han realizado diferentes estudios taxomanicos de algunas especies de la familia Cortinariacea. Hew ex Pourar. e inclusen algunos Cortinarius que Ituchlicin en lit rona de Vialdivia.

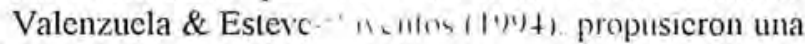
nueva especic de Cortumurims "r' hurakii) recogida en la Cordillera Pclada de Valdı ıa.

\section{MATERIAL Y METODOS}

Las prcparaciones microscópicas han sido realizadas en $\mathrm{KOH}$ al $5 \%$, rojo congo amoniacal y reaclivo de Melzer. Las microfolografias han sido realizadas en un microscopio Nikon modelo Labophot, con contraste de fase y sistema folográfico automático incorporado y en un microscopio de barrido Zeiss DSM-950 según la técnica indicada en Moreno et al. (1997). La película utilizada fue Kodak plus-x-pan 125 ASA.

El matcrial estudiado se conserva en el herbario del Departamento de Biología Vegetal (Botánica) de la Universidad de Alcalá (AH) y un duplicado en el herbario del primer autor en la Universidad Austral de Chile.

\section{RESULTADOS Y DISCUSION}

Cortinarius albocinctus Moser in Moser \& Horak, Beih. Nova Hedwigia 52:377 (1975). (Fig. la y Ib).

Material estudiado: En suelo, bajo Nothofagus dombeyi, Rebellín. Valdivia. 29-05-1990, leg. E. Valenzuela y J. Grinbergs. AH 14264. En humus de Nothof agus dombeyi con Saxegothea sp., Lago Frias, Prov. Río Negro, Argentina, 6-IV-1963, leg. M. Moser. Colección ZT 74/148. Typus $63 / 28+$ (IB).

Observaciones: Una descripción detallada de esta especie se aporta en Horak (I980). Cortinarius albocinctus se caracteriza por presentar un pilco de $2.5-5 \mathrm{~cm}$ de diám. convexo a umbonado-expandido, scco, higrófano, liso, ferruginoso a rojo castaño o más pálido al secarsc. Margen decurvado, en la juventud con restos de velo universal blanco, en la vejez con manchas blancas del velo, scdosasfibrilosas, que forman una banda característica. Estípite de 3-7 (9) × 0.2-1 cm de diám., claviforme, scco, glabro, blanco y en la vejez pálido sucio. Cortina y velo universal blanco. De su microscopía destacan sus esporas de 9-10.5 (12) $\times 5-5.5 \mu \mathrm{m}$, anchamente clipsoidalesaamigdaliformes, finamente verrugosas. Al microscopio clectrónico de barrido la ornamentación esta formada por verrugas que se anastomosan formando pequcñas crestas.

Una especie próxima a Cortinarius albocinctus, cs
C. nitcllinus Moser, el cual presenta basidiocarpos más robuslos y csporas subaniigdaliformes y subverrugosas.

C. albocinctus es una especie muy común, que fructifica en bosqucs de Nothofagus ( $N$. betuloides, $N$. dombeyi, N.obliqua y N.pumilio) mezclado con Saxegothaea sp. Ha sido citada por Moser \& Horak (1975), Horak (1980) y Lazo (1984). Se conoce de Argentina (Rio Negro, Valle Frías, Puerto Manzano, Nahuel Huapi, Neuquén y Tierra del Fuego) y Chile (Cerro Roble, Santiago, Curanilahue y Valdivia),

Cortinarius amoenus (Moser \& Horak) Moser \& Horak, Fl. Criptg. Tierra del Fuego 11 (6):484 (1980). (Fig. Ic). $=$ Dermocybe amoena Moser \& Horak, Beil. Nova Hedwigia 52:486 (1975).

= Dermocybe hypoxantha Horak in Moser \& Horak, Beil. Nova Hedwigia 52:510 (1975).

Material estudiado: En suelo, bajo Nothofugus dombeyi, a $50 \mathrm{Km}$. al Sur de Paillaco por la carretera Panamericana, Valdivia, 19-V-1992, leg. E. Valenzuela y S, Garnica, AH 15462.

Observaciones: Una descripción de esta especie viene indicada en Horak (1980). Podemos destacar de su macroscopía, su píleo de $2-6.5 \mathrm{~cm}$ de diám., convexo a anchamente umbonado-expandido, gelatinoso, no higrófano, con fibrillas innatas, de color castaño a castaño oscuro con tintes olivácco cástaños en el centro y margen castaño amarillento, estriado, cubicrto por fibrillas amarillentas en los ejemplares jóvenes. Láminas anexas a adnato-emarginadas a veces subdecurrentes, amarillas tornándose ocre ferruginosas. Estipite de 2-7 (12) x 0.3$0.7 \mathrm{~cm}$, (base hasta $1.5 \mathrm{~cm}$.) subclaviforme, ocasionalmente cilíndrico, seco, fibriloso, amarillo intenso, hacia la base con una faja subpersistente de velo castaño pálido y micelio amarillo. Carne amarillo castaño bajo la cuticula. De su microscopia destacamos sus esporas de 7-8.5 $\times 4.5$ $5.5 \mu \mathrm{m}$, ovoides a anchamente elipsoidales, verrugosas con la ornamentación más notoria hacia el ápice. $\mathrm{Al}$ microscopio electrónico de barrido la ornamentación esta formada por verrugas planas anastomosadas formando un pscudoretículo.

Especies próximas a Cortinarius amoenus son Dermocybe icterina Horak, cuyos basidiocarpos presentan un color amarillo intenso; pílco de $3-5 \mathrm{~cm}$ de diám., estipite dc $6-10 \times 0.4-1 \mathrm{~cm}$, el cual en la madurez es amarillo pardo y las esporas miden 7.5-8.5 $\times 4.8-5.4 \mu \mathrm{m}$. Cortinarius luteostriatulus es otra especie próxima que comentaremos más adelante.

Cortinarius amocnus es una especie micorrizógena de Nothofagus dombeyi, N. pumilio, N. betuloides y N. anturctica, ha sido citada de Argentina (Neuquén, Río 
Negroy Tierradel Fuego)y Chile(Rio Rubensy Magallanes) por Horak (1980).

Cortinarius austroacutus Moser in Moser \& Horak, Bcih. Nova Hcdwigia 52:287 (1975). (Fig. Id).

Material estudiado: En bosque de Nothofagus, cerea de Luma gayana, Rebellín, Valdivia, 17-V-1992, leg. E, Valenzuelay S. Garnica, AH 15535. Argentina, Tierra del Fucgo, Dpto. Ushuaia, Ticrra Mayor. Horak (ZT 74/213).

Observaciones: Una descripción de csta especie viene indicada en Horak (1980). Cortinarins austroacutus se caracteriza por su píleo muy pequeño de $0.5-1.5 \mathrm{~cm}$ de diám., cóníco a cónico-umbonado. higrófano, cstriado a fibriloso, de color castaño ocráceo, más pálido cuando seco, de margen decurvado, estriado. cubierto en la juventud con fibrillas blancas del velo. Líminas adnatas a anexas, ocráceo castaño con la arista cntera y concolora. Estipite de $2-5 \times 0.1-0.25 \mathrm{~cm}$, cilindrico, concoloro al pileo por debajo de la cortina, con fibrillas apretadas longitudinales blancas. Esporas de 6-7.5 (8) $x+-4.5 \mu \mathrm{m}$, anchamentc elipsoidales, verrugosas, las verrugas más notorias se situan hacia cl ápicc. Al microscopio clectrónico de barrido la ornamentación esla formada por verrugas pequeñas y laxas.

Una especic próxima a Cortinarius austroacutus es $C$. squamiger Moser, esta última especie se diferencia por presentar un pileo convevo-umbonado, amarillo pardo a rojizo, y por el pilco y estipite que presentan escuámulas de color blanco. C. austroacutus esta estrechamente relacionado con $C$. acutus Fr., esta última especie de acuerdo a Moser (1983), difiere por presentar queilocistidios y esporas de mayor tamaño. Gcográficamente C. austroucutus se distribuye en Chile (Valdivia a Magallanes) y Argentina (Tierra del Fuego), fructifica en suclos con Nothofagus nitida, $N$. betuloides y $N$. pumilio mezclados con Chusquea sp., Desfontaina espinosa y Blechnum sp.

Cortinarius austrolimonius Moser \& Horak, Beih. Nova Hedwigia 52:45+ (1975).

Material estudiado: En suclo, bajo Nothofugus dombeyi, Rebellín, Valdivia, 17-V-1992, lcg. E. Valcnzucla. AH 15463. En suelo, bajo Nothofugus dombeyt y N. pumilio, Los Troncos, Valdivia, 19-V-1992, Icg. E. Valcmzuclay S, Garnica, AH 15+64. En selva de Nothofugus (N. dombeyi y N. pumilio). Lago Frías, Prov. de Río Negro, Argentina, 21-III-1963, leg. M. Moser, Typus 63/112 (IB).

Observaciones: Una descripción de esta especic sc indica en Horak (1980). Cortinarius austrolimonius se caracte- riza macroscópicamente por presentar un píleo de 2.5-8. $\mathrm{cm}$ de diám., convevo a deprimido-convevo, castaño rojizo a castaño, en la vejez castaño grisáceo, seco, glabro, hacia el margen estriado e incurvado, ocasionalmente arrugado, cubicrto de diminutas escamas, con fibrillas lanosas del velo. Láminas emarginadas, apretadas, argiláceas, tornándose castaño amarillentas ferruginosas con la arista concolora. Estipite de $3.5-10 \times 0.6-1.5 \mathrm{~cm}$, cilindrico o subclaviforme, seco, fibriloso, amarillo o amarillento, por dcbajo de la cortina con varias fajas de color castaño rojizo deI velo, hacia la base con tomento blanco. De su microscopia destacan sus esporas de 6.5-8 (8.5) $\times 5-6(6.5) \mu \mathrm{m}$, subglobosas a anchamente clipsoidales, verrugosas sin placa suprahilar.

Una especic próxima a Cortinarius austrolimonius es $C$, teraturgus Moser, este presenta esporas amigdaliformes de 7.8-10.6 $\times 4.2-5.8 \mu \mathrm{m}$; pileo de color rojo a anaranjado pardo: velo rojizo pardo; láminas argiláceas, luego parduzcas con tonos amarillentos; pleuro y qucilocistidios.

C. austrolimonius es una especie micorrizógena que de acuerdo a Horak (1980), fructifica en forma cespitosa más raramente aislada en suclo, bajo Nothofugus dombeyi, N. betuloides y N. pumilio, tanto en Argentina (Río Negro y Tierra del Fuego) como en Chile (Valdivia). Garrido (1988) ha citado C. austrolimonius de Angol y Curanilahue, fructificando en los bosques antes indicados $y$ además en bosques de Nothofagus spp. mezclados con Weinmania trichosperma.

Cortinarius austrosalor Moser in Moser \& Horak, Beih. Nova Hedwigia 52:248 (1975).

Material estudiado: En suclo, bosque de Nothofagus dombeyi y N. pumilio, Rebcllín, Valdivia, 18-V-1990, lcg. E. Valenzucla y J. Grinbergs, AH 14288. En suelo, bajo Nothofagus dombeyi, Los Troncos, Valdivia, 19-V1992, leg. E. Valenzuela, AH 15291. Selva de Nothofagus (N. dombeyi, N. alpina y N. betuloides), Lago Frias, Prov. Ncuquen, Argentina, 4-IV-1963, leg. M. Moser, Holotypus 63/233 (IB).

Observaciones: Especie común de la zona valdiviana, caracterizada por su pílco de $5-9 \mathrm{~cm}$ de diám., convexo a irregularmentc umbonado-expandido, glutinoso, no estriado-pclúcido, de color violeta azul claro, hacia el disco cs algo fibriloso y más pálido, en la madurez pardo oscuro con tintes violctas. Láminas emarginadas, concoloras al pilco. Estípite de $7-15 \times 0.8-1.5 \mathrm{~cm}$, hacia la base claviforme de hasła $3.5 \mathrm{~cm}$ de diám., glutinoso, de color violcta pálido y hacia la base blanquecino. Restos de cortina ch la parte apical del estípite, de color blanquecino azul pálido. Carne espesa, blanca en el píleo, violáceo 
pálida hacia la base del estípite. Olor desagradable. fćtido. Esporas de 9-11 $\times 5-6(6.5) \mu \mathrm{m}$, anchamente clipsoidales a amigdaliformes, verrugosas. Basidios de $28-30 \times 8.5-10.5$ $\mu \mathrm{m}$, claviformes, tetraspóricos. Qucilocistidios de 25-3()$50 \times 4.5 \times 12 \mu \mathrm{m}$ cilíndricos a vesiculosos, con el ápice apendiculado a subcapitado.

Cortinarius austrosalor, es una especic micorrizógena que fructifica en bosques de Nothofugus ( $N$. dombeyi, N. alpina y $N$. betuloides con las especies antes citadas mezcladas con Chusquea coleau). fue primero citado en Argentina (Lago Frias, Prov. de Ncuquen) y luego en Chile (Cerro Mirador, Cordillera Pelada; Valdivia, Chiveria: La Unión. Pucrto Fao: Osorno) por Moser \& Horak (1975).

Cortinarius flammuloides Horak \& Moser, in Moser \& Horak, Beih. Nova Hedwigia 52: 185 (1975). (Fig, 1c).

Material estudiado: En suelo, bajo Nothof ugus dombeyi, Jardin de Anatomia, Universidad Austral de Chile, Valdivia, 16-05-1990. leg. E. Valenzucla, AH 13144y AH 14261. En suclo, bajo Podocarpus saligna, Jardín Botánico Universidad Austral de Chile, Valdivia, 22-V-1992, leg. E. Valenzucla, AH 15292. En suclo, bajo Nothofugus pumilio, La Paloma, Valdivia, 19-V-1992. Icg. E, Valenzucla y S. Garnica, AH 15457. En suclo, selva de Nothofagus ( $N$. dombeyi y N. antarctica), Valle Frias, "La Panque". Prov. Ncuquén. Argentina, leg. E. Horak, Holotypus 518 (IB). Estancia Nueva Argentina, Tierra del Fucgo, Dpto. Río Grande, Argentina, leg. Singer, Typus (M-333) (IB). Colecciones Dr. Horak. 74/88, 74/I62 (ZT).

Observaciones: Una descripción detallada de esta especic sc indica en Horak (1980). De su macroscopía destacan su pilco de 2.2-8 $(8.5) \mathrm{cm}$ de diám., convexo a umbonadoexpandido, gelatinoso. castaño amarillento a castaño anaranjado, con el margen estriado, con restos amarillos del velo universal. Láminaș adnatas a emarginadas, amarillo pálidas. luego ferruginoso amarillentas, con la arista concolora. Estipite de $4.5-6.5 \times 0.4-1.1 \mathrm{~cm}$, cilindrico con la base bulbosa, fibriloso, blanquecino amarillento, hacia abajo amarillo, con cortina subpersistente formando numerosas escuámulas fibrilosas o fajas incompletas. base del estipite cubierta de micelio amarillo que perdura en las muestras de herbario. De su microscopia sobresalen sus esporas de (8) 9-11 $\times$ 5.5-6 $\mu \mathrm{m}$. sublimoniformes con mucrón neto, verrugosas, dextrinoides sobre todo las verrugas. Al microscopio electrónico de barrido la ornamentación esta formada por verrugas que se unen formando crestas de longitud variable.

Una especie próxima a Cortinarius flammuloides es $C$. obrusseus Horak. el cual presenta un estípitc cilindrico igual o de base atenuada: píleo de $3-5.5 \mathrm{~cm}$ de diaim amarillo pardo pálido; esporas de 8-9.5 x 4.5-5.5 $\mu \mathrm{m}$, sublimoniformes a amigdaliformes y débilmente asperuladas.

Cortinarius flammuloides es una especie micorrizógena, que de acuerdo a Horak (1980) fructifica en bosque de Nothofagus ( $N$. dombeyi, $N$. antarctica, $N$. oblicua, $N$. pumilio y $N$. betuloides), por su parte Garrido (1988). scĩala que C. flammuloides, fructifica entrela VII y IXRegión de Chile principalmente bajo $N$. dombeyiy $N$. obliqua mezclado con Aristotelia chilensis y Fuchsia magellanica, de acucrdo con este mismo autor $C$. flammuloides, se distribuye en Chile desde Angol hasta Llanquilue $y$ en Argentina de Neuquen a Tierra del Fuego.

Cortinarius fuscus Moser in Moser \& Horak, Beih Nova Hedwigia 52:349 (1975), (Fig. If).

Material estudiado: En suclo, en bosquede Austrocedrus chilensis, Parque SAVAL, Valdivia 13-V-1992, leg. S. Garnica y E. Valenzucla, AH 15460 .

Macroscopía: Pílco de $0.5-2 \mathrm{~cm}$ de diám., campanulado en la juventud, lucgo conveso umbonado. Cutícula higrólana, lisa, de color chocolate pardo oscuro. Margen no estriado, concoloro a la cutícula o un poco más pálido, Láminas adnato-emarginadas, en la juventud de color amarillo oscuro finalmente castaño ferruginosas. Arista débilmentc àserrada, concolora a la lámina. Estipite de 3$4 x(0.2-0.3(0.5) \mathrm{cm}$, cilindrico, un poco atenuado hacia el ápicc, seco. pardo blanquecino sucio, hacia $\mathrm{cl}$ ápice con tintes grisácco lilacinos. Cortina blanca en la juventud. Olor y sabor no apreciables. El pilco y la carne con $\mathrm{KOH}\left(\mathrm{I}\left(0^{\circ} \%\right)\right.$ ), se tornan de color pardo negruzco pálido. Microscopía: Esporas de 6.5-7.5 x +-4.8 (5) $\mu \mathrm{m}$, anchamente elipsoidales, asperulado-verrugosas, la ornamentación es más acentuada hacia el ápice. Al microscopio electrónico de barrido la ornamentación esta formada por verrugas densas de ápice plano que se unen en crestas de longitud variable. Basidios de $27-30 \times 7-8$ $\mu \mathrm{m}$, claviformes a cilindricos cortos, tetraspóricos, híalinos. Cistidios nulos. Pileipellis formada por hifas de 3-7 (8) $\mu \mathrm{m}$ de diám.. hialinas. Fíbulas presentes.

Observaciones: Cortinarius fuscus, se cita por primera vez para Chile. Moser \& Horak (1975), indican que esta especie fructifica en bosque de Nothofagus dombeyi mezclado con Saxegothea sp., en Argentina (PuertoFrias, Prov. de Rio Negro), estos mismos autores señalan que $C$. lignyotus Horak es una especie próxima a $C$. fuscus, el cual presenta un pilco de $1-1.5 \mathrm{~cm}$ de diám, cónicocampanulado, pardo-negro oscuro; margen estriado: láminas pardas oscuras con la arista ascrrada blanca; 
estipitc concoloroal pilco, cubierto con restos fibrilosos de velo blanco y esporas de 7-8 $\times 5-5,6 \mu \mathrm{m}$.

Cortinarius horakii Valenzucla \& Esterc-Raventós Mycol. Res. 98:937-938 (1994.) (Fig. 2a-g).

Material estudiado: En suclo, bajo Nothofagus dombeyi, Cordillera Pclada. Valdivia, 2+-1V-1992. Icg. H. Peredoy S. Garnica, AH 15535. En suclo, bajo Nethof fugus dombeyi, Cordillera Pclada. 2+-IV-1992, Ieg. H. Pcredo. AH 15536.

Macroscopía: Píleo de $3.5-10.5 \mathrm{~cm}$ de diám., convero a plano convero, a veces subumbonado. Cuticula víscida. subhigrófana. glabra, no csiriada. de color pardo más pálido hacia el margen. Margen decurvado, entero, cubierto de restos de velo blanco en la juventud. Láminas adnatas. ventrudas. apretadas. pardo pálidas a pardo crémcas. en la vejee ferruginosas. con la arista concolora. Estipite de $4.5-9.5 \times 1-1.5 \mathrm{~cm}$. la base de $2.2-2.5 \mathrm{~cm}$. claviforme. seco, de color pardo crémco a pardo pálido, cubierto de fibrillas longitudinales del velo de color pardo oscuro. Carne blanquecina crémca, parda Jajo la culícula del pílco. dccolorándose a pardo pálido cn la vẹjc\%. Sabor y olor fúngicos, Reacción con $\mathrm{KOH}(\mathrm{I} 0 \%)$ sobre cl pílco. sc torna pardo-amarillento. en la carne negativa.

Microscopía: Esporas de $6.5-8 x+-4.5(-5) \mu m$. anchamente clipsoidales a subamigdaliformes, finamente verrugosas, cuyo ápice presenta al microscopio óplico un pseudocallo similar a un poro germinativo. Al microscopio electrónico de barrido. las esporas muestran en su ápice una ornamentación más densa que scmẹja a una especie de "caperuza"; esta formación csta conslituida por la aglomeración de verrugas del perisporio. dando una apariencia subuniforme; cn algunos casos ocurre como si esta caperuza se desvanecicra quedando cl ápice truncado; el resto de la pared esporal, presenta una típica ornamentación con verrugas densas, no excesivamente altas. y $\mathrm{cn}$ algunas zonas unidas irrcgularmentc, dando la apariencia de un subretículo. Basidios de 28-37 (-42) $\times 7$ $8 \mu \mathrm{m}$. claviformes, tetraspóricos, hialinos o con un contenido amarillento pálido en $\mathrm{KOH}(10 \%)$. Queilocistidios de $25-30 \times 3-5 \mu \mathrm{m}$. hialinos. cilíndricos. con un largo o corto cucllo recurvado y atenuándose hacia $\mathrm{cl}$ ápice. de modo semcejante al cucllo de un cisne; hacia la parte basal presentan de 2 a 3 tabiques y una fibula en su basc. Pilcipellis formada por hifas cilindricas de $t-7 \mu m$ de diám. con pigmento incrustantc amarillento-pardo en $\mathrm{KOH}(10 \%)$, en una matriz mucilaginosa. Fibulas presentes.

Observaciones: Cortinarius horakii sc caracteriza microscópicamente por prescntar csporas clipsoidales a subamigdaliformes, las cuales presentan en el ápice un pscudocallo similar a un poro germinativo $y$, sus queilocistidios cilindricos con un largo o corto cuello recurvado similar al cucllo de un cisne, que muestran hacia su base 2 a 3 tabiques. Macroscópicamente resaltan: su pílco víscido, pardo. con $\mathrm{cl}$ margen más pálido, en la jurentud cubicrto por restos de velo de color blanco, y su estípite claviforme de color crémeo-pardo, cubierto con fibrillas longitudinales marrones oscuras.

Una cspecic próxima a $C$. horakii es $C$. albobrunneus Moser, que de acuerdo a su autor, presenta su pílco castaño a anaranjado-pardo (similar a Tricholoma albobrunneum). cstipitc bulboso a claviforme. esporas amigdaliformes a sublimoniformes y queilocistidios lageniformes a fusiformes.

Cortinarius lebre Garrido, Bibl. Mycol, 120:153(1988). (Fig. 3a).

Material estudiado: En suelo, bajo Nothofagus obliqua, Limite norte entre Valdivia y Temuco, 3-VII-1990, leg. M. Jeria, AH $15+67$.

Macroscopía: Pílco de $4-15$ (20) cm de diảm., convexo a plano-umbonado. ligeramente viscido. liso, finamente fibroso, de color vinácco en la juventud, finalmente ocre pardo con tonos lilacinos. Margen involuto cn la juventud, luego decurvado algo ondulado o plano y roto con restos dc velo submembranoso blanco. Láminas ancias a adnatoemarginadas, gruesas, apretadas, pajizas en la juventud, luego ocráceo-pardas. frecuentemente con pequeñas manchas de color rojo-pardo. Estipite de $6-15 \times 1.5-3 \mathrm{~cm}$, sólido, cilindrico o atcnuándose liacia la base, seco, finamente fibriloso-cscuamuloso, blanquecino con tonos rọoparduzcos. Vclo fibriloso, blanco. formando zonas anulares incompletas o completas sobre el estípitc. Carne fibrosa. blanquecina. Olor a "madriguera de liebre" o como Tuber magnatum. Sabor a "agarico" (Agaricus hisporus). El pílco y estipite, se tornan de color rojo parduzco con $\mathrm{KOH}(10 \%)$.

Microscopia: Esporas de 8-il (13) x 5-7 $\mu \mathrm{m}$, anchamente clipsoidales a amigdaliformes, verrugosas. Al microscopio electrónico de barrido la ornamentación esta formada por verrugas poco marcadas y densas que forman crestas de longitud variable. Basidios de 26-40 $\times 6-10 \mu \mathrm{m}$, claviformes, tetraspóricos, hialinos. Cistidios nulos. Pilcipellis formada por hifas cilindricas de $2-7 \mu \mathrm{m}$ de diám., hialinas o con pigmento no incrustante de color rojo-pardo, en matriz mucilaginosa. Fibulas presentes.

Observaciones: Especic chilena, citada como nueva para laciencia por Garrido (1988), en Concepción. Cortinarius 
lebre, fructifica formando pequeños grupos en bosques de Nothofagus (N. obliqua, N. antarctica y $N$. glauca mezclados con Gomortega keule), cstc mismo autor indica que este hongo es muy apreciado y que se consume por la población indigena chilena.

Cortinarius luteostriatulus (Moser \& Horak) Valenzuela \& Moreno. Bol. Soc. Micol. Madrid 19:289. (1994). (Figura 3b)

$=$ Dermocybe luteostriatula Moser \& Horak. Beih. Nova Hedwigia 52: 501 (1975).

Material estudiado: En suelo. bạjo Nothofugus obliqua y $N$. dombeyi, Popoćn, San Juan de la Costa. Osorno. I1V-1992, leg. S. Garnica, AH 15465.

Macroscopía: Pilco de $1.5-4 \mathrm{~cm}$ de diám., primero semigloboso. lucgo campanulado. en la vejez convexoumbonado. estriado por transparencia, amarillo-anaranjado en el disco, hacia el margen amarillo brillante con tintes oliváceos. en la vejez amarillo-anaranjado a amarillo-pardo pálido uniforme. Margen cstriado amarillo brillante a amarillo vitclino. Láminas adnato-cmarginadas, subdistantes. de color anarillo limón, finalmente anaranjadas a amarillo-ferruginosas. arista subaserrada, y concolora a la lámina. Estipite amarillo de 5-8 $\times(0.2-0.4$ $\mathrm{cm}$, hacia la base mide hasta $0.7 \mathrm{~cm}$ de diám, cilindrico a claviforme. hacia la base amarillo-anaranjado a amariIlo-pardo pálido. Cortina amarilla. subpersistente hacia cl ápice del estípitc. tornándose pardo-anaranjada por las esporas. Carne amarilla, hacia cl pilco, pardo anaranjada con tonos oliváccos. Olor nulo. Sabor levemente rafanoide. El pileo y la carne con $\mathrm{KOH}(10 \%)$ se torna de color rojopardo.

Microscopía: Esporas de $6-8$ (9) $\times 4.2-4.8$ (5.2) $\mu \mathrm{m}$. anchamente clipsoidales a subanigdaliformes. verrugosas. AI MEB. la ornamentación csta formada por loscas y densas verrugas planas. Basidios de $25-35 \times 7-9.5 \mu \mathrm{m}$, claviformes. hialinos. Cislidios nulos a escasos e inconspicuos de $30-40$ × $6-7$ (8) $\mu \mathrm{m}$. cilindricos-claviformes. Pilcipellis formada por hifas cilindricas de 5-9 $\mu \mathrm{m}$ de diámetro, hialinas o con pigmento incrustante en las paredes anarillo-pardo en $\mathrm{KOH}(10 \%)$. Fibulas presentes,

Observaciones: Cortinarius luteostriatulus, se citó por primera vez. para Chile (Antillanca: Osorno) por Moser \& Horak (1975), esta especic sc conoce tambićn en Argentina (Valle Frias; Prov: de Rio Negro). Fructifica en bosques de Nothofagus dombey, N. pumilio y $N$ antarctica. C. luteostriatulus se caracteriza por su pilco estriado de la mitad hacia el margen, de color amarillopardo con tintes oliváccos. el margen amarillo brillante a amarillo vitelino, sus láminas amarillo limón a anaranjado-amarillentas y finalmente anaranjado-parduzcas; estipite amarillo y esporas de $6-8(9) \times 4-4.8(5.2) \mu \mathrm{m}$.

Una cspecie a próxima es Cortinarius flavofuscatus Horak \& Moser, que se diferencia por poseer esporas más anchas $7.5-8.5 \times 5.2-6.5 \mu \mathrm{m}$, pilco amarillento-pardo con el centro pardo oscuro con tintcsoliváccos, débilmente cstriado por transparencia, láminas amarillentas tornándose en la vejez naranja pardo y estipite amarillo.

Cortinarius magellanicus Speg., Bol, Acad. Nac. Ci. Córdoba 11:15 (1887). (Fig. 3c).

= Cortinarius aiacapia e Speg.. Bol. Acad. Nac, Ci. Córdoba 11:152 (1887).

= Cortinarius capitellinus Horak in Moser \& Horak, Beih. Nova Hedwigia 52:260 (1975).

Material estudiado: En suclo, bajo Nothofugus procera, Cordillera Pclada, Valdivia, 21-IV-1991, lcg. H. Pcredo, AH $15+59$. En jardines con Nothofugus dombeyi, Casa de Extensión, Universidad Austral de Chilc. Valdivia, 12V-1992, leg. E. Valenzucla. AH 15458. Puerto Harberton, Argentina, Ieg. I, Gamundi (LPS 35384). Colecciones (BAFC 23317. BAFC 23258, BAFC 2326). Tierra Mayor, Argentina, Colecciones ZT 74/91: ZT 74/191, ZT TF 50 Hololypus Cortinarius capitellinus Horak.

Observaciones: Una descripción detallada de esta especie sc indica en Horak (1980). Cortinarius magellanicus se caractcriza por su pilco de $1-8 \mathrm{~cm}$ de diám.. convevo a umbonado-cxpandido. Cutícula glutinosa, finamente esiriada. lilacina, violácea a purpúrea en la juycntud. castaño ocrícca en la vejè. Láminas adnato-cmarginadas, lilácco pálidas en la juventud. finalmente castaño ferruginosas claras con la arista crenulada a fimbriada y concolora. Estípite de $3-12 \times 0.25-1(1.8) \mathrm{cm}$. cilíndrico a subclaviforme. cl ápice concoloro al pilco, hacia la base blanquecino a castaño pálido. glutinoso. Cortina fibrilosa, pobremente desarrollada. debajo de la cortina con un reicstimicnto glutinoso grueso que en los ejemplares jóvenes esta unido con el margen del pilco. En condiciones de sequedad cl mucilago forma un anillo membranoso, persistcutc. con cstrias longitudinales. Esporas de 9-12.5 x 5-7 $\mu \mathrm{m}$, anchamente clipsoidalcs, verrugosas, las verrugas más toscis se presentan hacia $\mathrm{cl}$ ápice. Al MEB, la ornamentación csta formada por verrugas planas toscas, densas. que sc anastomosan dando una aparicncia subreticulada. Qucilocistidios de 201-55 × 7-16 $\mu \mathrm{m}$. claviformes. subcilindricos o fusiformes, de paredes delgadas, hialinos. $\mathrm{en} \mathrm{KOH}(10 \%)$ con pigmento plasmático de color lílácco.

Cortinarius magellamicus, es una especic micorrizógena. que fructifica en forma cespitosa en bos- 


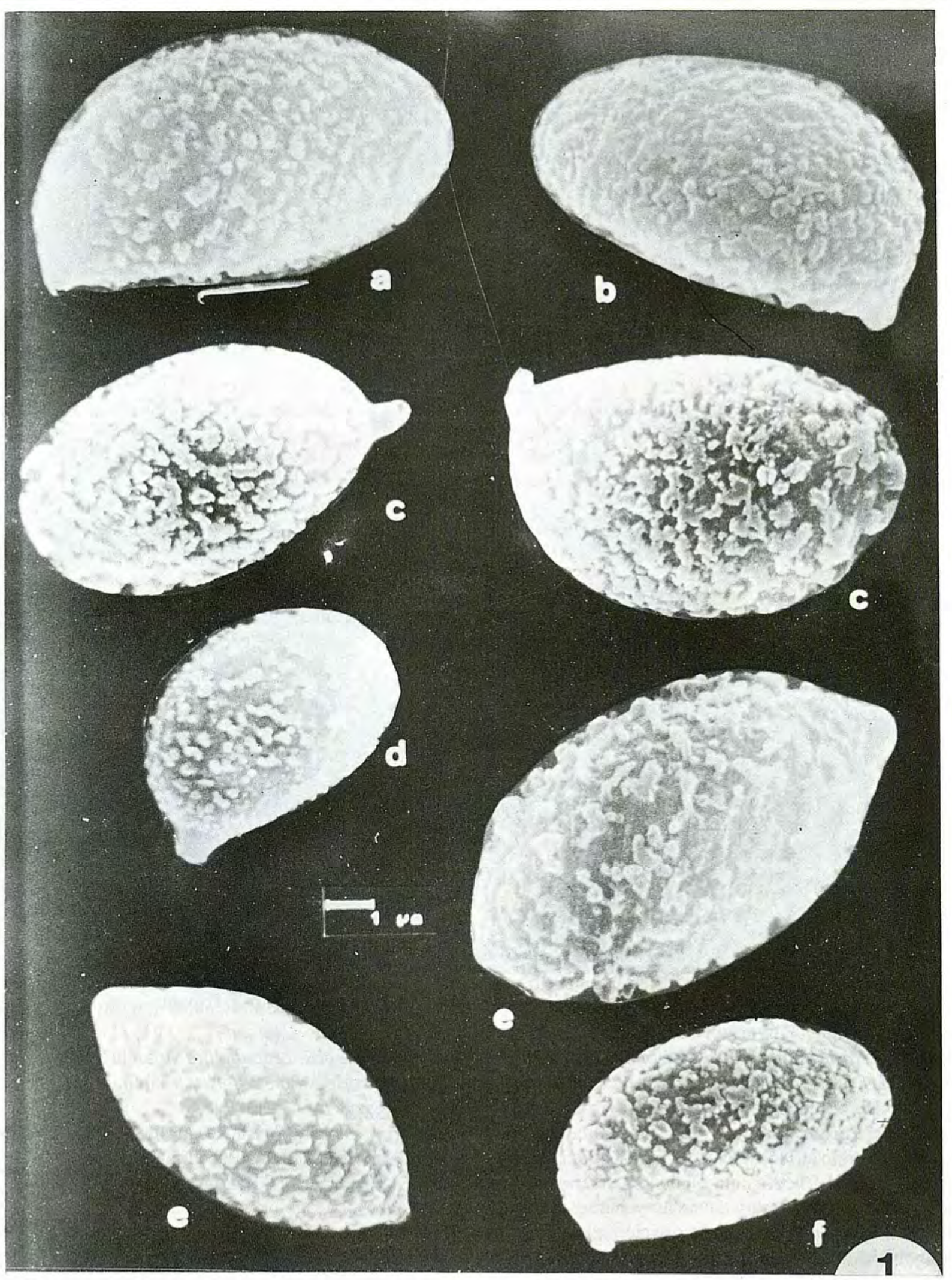

Fig 1.- a Cortinarius albocinctus (AH 14264), b. C. albocinctus (IB 63/284 tipo); c. C. amoenus (AH 15462); d. C. austroacutus (AH 15535); e. C. flammuloides (AH 14261); f. C. fuscus (AH 15460) 


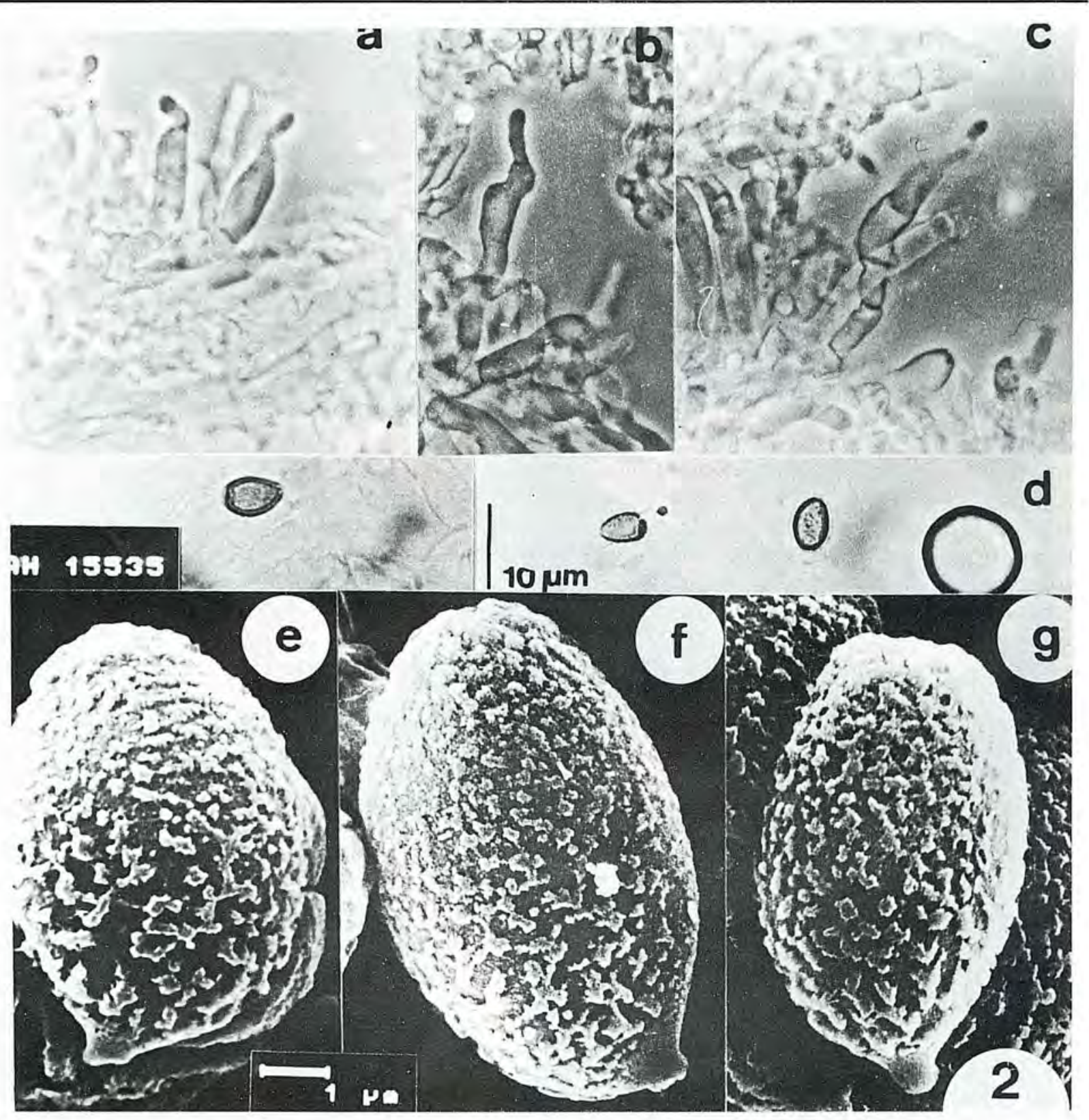

Fig 2.- Cortinarius horakii (AH 15535). a-c. Queilocistidios, d-g. Esporas.

ques de Nothofagus (N. pumilio, N. dombeyi, N. betuloides, $N$. nitida y $N$. antarctica), de acuerdo a Horak (1980), se conoce de Argentina (Neuquén, Río negro y Tierra del Fuego) y de Chile (Magallanes).

Garrido $(1985,1988)$, nos indica que $C$. magellanicus también fructifica en Chile con Fitzroya, Pilgerodendro y sobre restos de hojas y humus de Fagus antarctica, ampliando su distribución a Concepción, Angol, Valdivia, Osorno, Punta Arenas y Puerto Aysen.

Cortinarius olivaceobubalinus (Moser) Valenzuela \& Moreno. Bol.Soc.Micol. Madrid 20: 194 (1995). (Fig. 3d).
= Dermocybe olivaceobubalina Moser in Moser \& Horak, Beih. Nova Hedwigia 52:496 (1975).

Material estudiado: En bosque de Nothofagus spp., mezclado con 'Temu", Rebellín, Valdivia, 17-V-1992, leg. E. Valenzuela y S. Garnica, AH 15466.

Macroscopía: Píleo de 2-2,5 cm de diám., convexo a obtuso-cónico a subumbonado, seco, estriado por transparencia cuando húmedo, de color oliváceo pardo oscuro en el disco, hacia el margen oliva amarillento a oliva-pardo pálido. Margen cstriado, concoloro al píleo. Láminas 
cmarginado-adnatas, subdistantes, verde-amarillentas claras, en la madurez verde-ferruginosas. Arista débilmente aserrada, concolora a la lámina. Estipite de 3-5 $\mathrm{x}$ $0.2-(), 4 \mathrm{~cm}$, cilíndrico, de color oliva-ocre a oliva-amariIlento, hacia la base oliva pardo. Carne olivácco pálida. hacia el pílco y base del eslípile parclo pálido. Olor y sabor fúngico.

Microscopía: Esporas de 6-8.5 (9) $\times$ 4-5 (5.5) $\mu \mathrm{m}$, ferruginosas, anchamente elipsoidales, desde casi lisas a débilmente verrugosas. Al microscopio electrónico de barrido la ornamentación csta formada por verrugas que forman pequeñas crestas. Basidios de 20-30 $\times 6-8(10) \mu \mathrm{m}$, claviformes a cilíndricos, tetraspóricos, hialinos. Cistidios nulos. Pileipellis formada por hifas cilindricas de $3-10 \mu \mathrm{m}$ de diám, con pigmento membranoso de color pardo en $\mathrm{KOH}(10 \%)$. Fíbulas presentes.

Observaciones: Especie bastante común y citada en Chile (Cerro Mirador y Cordillera Pelada) según Moser \& Horak (1975). fructilica en bosques de Nothofagus (N. dombeyi, N. nitida, N. proceray $N$. anturctica), mezclado con Podocarpus mubigenus, Weinmannia sp.. Laurelia $\mathrm{sp}$, Saxcgothea sp, Blechmum sp. y Chusquea montana. La estirpe Olivaceobubalina, presenta además de Cortinarius olivaceobubalinus, dos especies más, en las que el color predominante del pílco cs pardo, el primero de ellos Dermocybe semipellucida Moser, presenta un píleo de $1.8-3.5 \mathrm{~cm}$ de diám, castaño pardo, con un pcqueño unbo, estriado por transparencia: láminas ocrácco pardo a pardo; cstipitc gris pardo pálido; csporas de 7-9.5 (10.2) $\times 4.4-5.3 \mu \mathrm{m}$ : la segunda Dermocybe corymecystis Moser. sc caracteriza por su pilco de color pardo con tintes oliváccos, amarillento-rojizo parduzcos en la madurez. fibriloso a glabro de $2-4.5 \mathrm{~cm}$ de diám; láminas amarillentas con tintes verdosos. tornándosc oliváceas y fimalmente amarillo-pardo-rojizas; cstipite fibriloso amarillento verdoso y esporas de $6.6-8 \times 3.5-4.5 \mu \mathrm{m}$.

Cortinarius pachynemeus Moser \& Horak, Bcil. Nova Hedwigia 52:313 (1975). (Fig. 3c).

Material estudiado: En suclo, bajo Nothofugus dombeyi, Rcbcllíi. Valdivia, 17-V-10)2. Icg. E. Valcmsucla, AH 15+75. En suclo. bajo Nothofugus pumilio, Antillanca. Osorno, 26-V-1997, Icg. E. Valenzucla. AH 195()2.

Macroscopía: Pilco de 1-2 cm de diám., convero-umbonado, higrófano, rojo-pardo oscuro. hạcia cl margen más pálido, cuando seco de color ocrc. Margen pelúcidoestriado, en la juventud con restos de velo blanco, que se torna de color castaño a ocrácco. Láminas cmarginadoadnatas a ancxas. de color ferruginoso. Estipite de 1.5-3x
$0.1-(0.3 \mathrm{~cm}$, (hacia la base de $0.2-(0.6 \mathrm{~cm})$, cilindrico a claviforme-subfusiforme, algo cstriado, blanquecino en la juventud, lucgo blanco avellana. Carne escasa, blanca, en el estípitc blanco avellana. Olor y sabor no apreciables.

Microscopia: Esporas de 7-9x+-5 $\mu \mathrm{m}$, ferruginosas, anchamente elipsoidales, lisas a débilmente verrugosas hacia la parte apical. Al microscopio clectrónico de barrido la ornamentación csta formada por pequeñas verrugas esparcidas. Basidios de 25-3()x+-5 $\mu \mathrm{m}$, claviformes, tetraspóricos, hialinos. Cistidios nulos. Pileipellis formada por hifas cilindricas de $5-12 \mu \mathrm{m}$ de diám, hialinas o con pigmento incrustantc amarillo pardo en $\mathrm{KOH}(10 \%)$. Fibulas presentes.

Observaciones: Cortinarius pachynemeus, es una especic que sólo se conoce y ha sido citada en dos oportunidades de Chile (Cuesta Santa Elvira, Valdivia y Cerro Roblc, Santiago) por Moser (Moser \& Horak, 1975), y fructifica en bosques de Nothofagus obliqua y $N$. obliqua var. macrocarpa.

Cortinarius pachynemeus, se caracteriza por su pequeño porte y sus esporas sublisas ", una especie próxima cs $C$. eluchus Moscr, cuyo pilco presenta colores más oscuros y esporas de $8-9,5 \times 5-5.5 \mu \mathrm{mI}$. clipsoidales y débilmente asperuladiss.

Cortinurius pugionipes Moser in Moser \& Horak, Bcil. Nova Hedwigia 52:1+6 (1975). (Fig. 3f).

Material estudiado: En suelo, bajo Nothof agus dombeyi, Rebellin, Valdivia. 17-V-1992, leg. E. Valenzuela, AH 15293 y AH 15+55. En suclo, bajo Nothofagus procera, Rcbellin. Valdivia, 18-V-1992. icg. E. Valenzucla y J. Grinbergs AH 15468. En suclo, bajo Nothofaguspumilio, Cerro Corlinario. Nahuci Huapi. Prov. Neuquen. Argentina. 16-III-1963, leg. M. Moser. Typus 63/72 (IB).

Macroscopía: Pílco de 6-12 (15) cm de diám., en la juventud hemisférico, luego convexo. glutinoso, de col or blanquecino-cremco en la juventud, luego ocráceo pálido en cl disco y hacia el margen crémco sucio, en la madurez ocráce pálido con el disco más oșcuro. Margen decurvado, fibriloso en la juventud por los restos de cortina de color blanco. Láminas enarginadas, densas, ventrudas, blancas en la jurcntud, luego ocre-anarillentas pálidas y en la vçez ocre muy tenue. Estipite de $10-18 \times 1-2.5 \mathrm{~cm}$, cilindrico, hacia la base atcnuándose y radicante, glutinoso. de color blanco, con restos del velo universal a modo de bandas ocráceas, en la madurez de color crema sucio seco, frecuentemente sólo con restos de velo a modo de pequeños copos. Carne cspesa. blanca. Olor nulo. Sabor poco remarcable. 
Microscopía: Esporas de 6.5-8 (9) $\times 4-5 \mu m$. anchamente elipsoidales a ovoide-elipsoidales, verrugoso-asperuladas. de color pardo-amarillo pálido. Al microscopio clectrónico la ornamentación esta formada por pequeñas verrugas densas apretadas entre sí. Basidios de 25-30 $\times$ 7-8.5 $\mu \mathrm{m}$. claviformes, tetraspóricos. hialinos. Cistídios nulos. Pilcipellis formada por hifas cilindricas de 2-5 (9) $\mu \mathrm{m}$ de diám.. en una matriz mucilaginosa. Fibulas prescntcs.

Observaciones: Cortinarius pugionipes, es una especic micorrizógena, que fructifica de forma fasciculada cn bosques de Nothofagus ( $N$. pumilio, $N$. dombeyi y $N$. procera), esta especic se cita por primera vez para Chile. Moser \& Horak (1975), la habian citado anteriormente desde Argentina (Nahucl Huapi. Cerro corlinario). Las recolecciones de Valdivia. corresponden macroy microscópicamente. así como la ecología, con el matcrial tipo revisado.

De acuerdo a Moser \& Horak (1975), se pucden considerar como especics próximas a Cortinarius pugionipes, C, cervinus Moser, C. longicaudus Moser y muy estrechamente relacionada, la varicdad $C$. pugionipes var. azonatus; la primera, presenta un pilco de hasta $20 \mathrm{~cm}$ de diám., amarillo-pardo en la juscntud y rojizo pálido en la madurcz: estípite blanco y profundanente radicante; csporas de 8,5-1) $\times 4.2-5 \mu \mathrm{m}$. C. Iongicaudus, posce píleo más pequeño de $2-6 \mathrm{~cm}$ de diám., blancoamarillento a ocrácco pálido: cstípitc cilíndrico blanco; csporas de 5-6 $\times 3,5-4 \mu m$ y no fructifica de forma fasciculada. Por último C. pugionipes var. azonatus, difiere de la especic tipo, por carecer el cstípite de bandas de velo, colores del píleo más claros y presentar queilocistidios.

Cortinarius rubrobasalis Moser \& Horak, Beil. Nova Hedwigia 52:442 (1975).

Material estudiado: En suclo, entre hojarasca y orilla de senderos de bosque de Nothofagus spp.. Rebellin, Valdivia, 29-V-1990, leg. E. Valcnzucla y J. Grinbergs, ÄH 152\%5. En suelo, bajo Nothofagus pumilio, Rcbellin, Valdivia, 17-V-1992, leg. E. Valenzucla, AH 15295. Selva de Nothofugus (N. dombeyi, $N$. pumilio y $N$. antarctica). Río Negro. Valle Frias, Argentina, 2t-III1963, leg. M. Moser. Tipo IB 63/173 (IB). En suclo. bajo Nothofagus pumilio, Cerro Cortinario Prov. Neuquen, Argentina. 5-IV-1963. lcg. M. Moscr, Isolypus IB 63/68 (IB).

Observaciones: Una descripción de csta especie se indica en Horak (1980). Cortinarius rubrobasalis se caracteriza por presentar un pilco de $2.5-6 \mathrm{~cm}$ de diám., convexo a anchamente umbonado-cxpandido. castaño-roji a a castaño oscuro cuando húmedo, densamente cubierto por fibrillas de color-anaranjado rojizas. Láminas adnatas a cmarginadas, ventricosas, lilacinas cuando jóvenes, tornándose ocráceas ferruginosas en la madurez, la arista es lisa y concolora. Estipite de $3-7 \times 0.5-1.5(2) \mathrm{cm}$, cilindrico a subfusiforme o claviforme, castaño claro hacia el ápice, por debajo de la cortina castaño-rojizo o castaño oscuro, cortina fibrilosa. persistente, hacia la base con 1-4 zonas de velo incompletas, fibrilosas, persistente. micelio basal amarillo. Esporas de 8-11 $\times 5-6.5 \mu \mathrm{m}$, anchamente clipsoidales a ovales, verrugosas, las verrugas más marcadas hacia el ápicc. Pilcipcllis formada por una cutis de hifas cilíndricas de 5-9 $\mu \mathrm{m}$ de diám., con pigmento plasmático c incrustante rojo víolácco en $\mathrm{KOH} 10 \%$.

Especic muy común y abundante en los bosques de Nothofagus ( $N$. dombeyi, N. pumilio y $N$. antarctica), también se le sucle encontrar fructificando en bosques de Fitzroya y Saxcgothea. Sc conoce en Argentina (Rio Negro, Ncuquén, Ushuaia y Tierra del Fuego) y en Chile (Antillanca, Los Robles. Puerto Natales, Ticrra del Fuego y Prov de Magallanes) según Moser \& Horak (1975) y Horak (1980).

Otras especies curopeas que de algún modo cstarian relacionadas con Cortinarius rubrobasalis, pero que dificren por su ccologia y características macro y microscópicas son $C$. bulliardii Fr. y $C$. erythroionipus Fay. Además Cortinarius hasirubescens Clel. et Harris, cl cual fructifica bajo Eucalyytus spp., en Australia.

\section{NUEVAS COMBINACIONES PROPUESTAS:}

Cortinarius amoenus (Moser \& Horak) Valenzuela \& G. Moreno comb. nov.

= Dermocybe amoena Moser \& Horak in Moser \& Horak. Bcill. Nova Hedwigia 52: 486 (1975).

Cortinarius flavofucatus (Moscr \& Horak) Valenzuela \& G. Moreno comb. nov:

= Dermocybe flavofucata Moser \& Horak in Moser \& Horak, Beih. Nova Hcdwigia 52:503 (1975).

Cortinarius icterinus (Horak). G. Moreno \& Valenzuela comb. nov.

= Dermocybe icterina Horak in Moser \& Horak, Bcih. Nova Hedwigia 52:584 (1975).

Cortinarius nuluelbutensis (Garrido)Valcnzuela \& G. Moreno comb, nov,

$=$ Dermocybe nahuelhutensis Garrido Biblioth. Mycol. 120:167 (1988).

Cortinarius oliveoicterinus (Moser) Valenzuela \& G. Moreno comb. nov. 

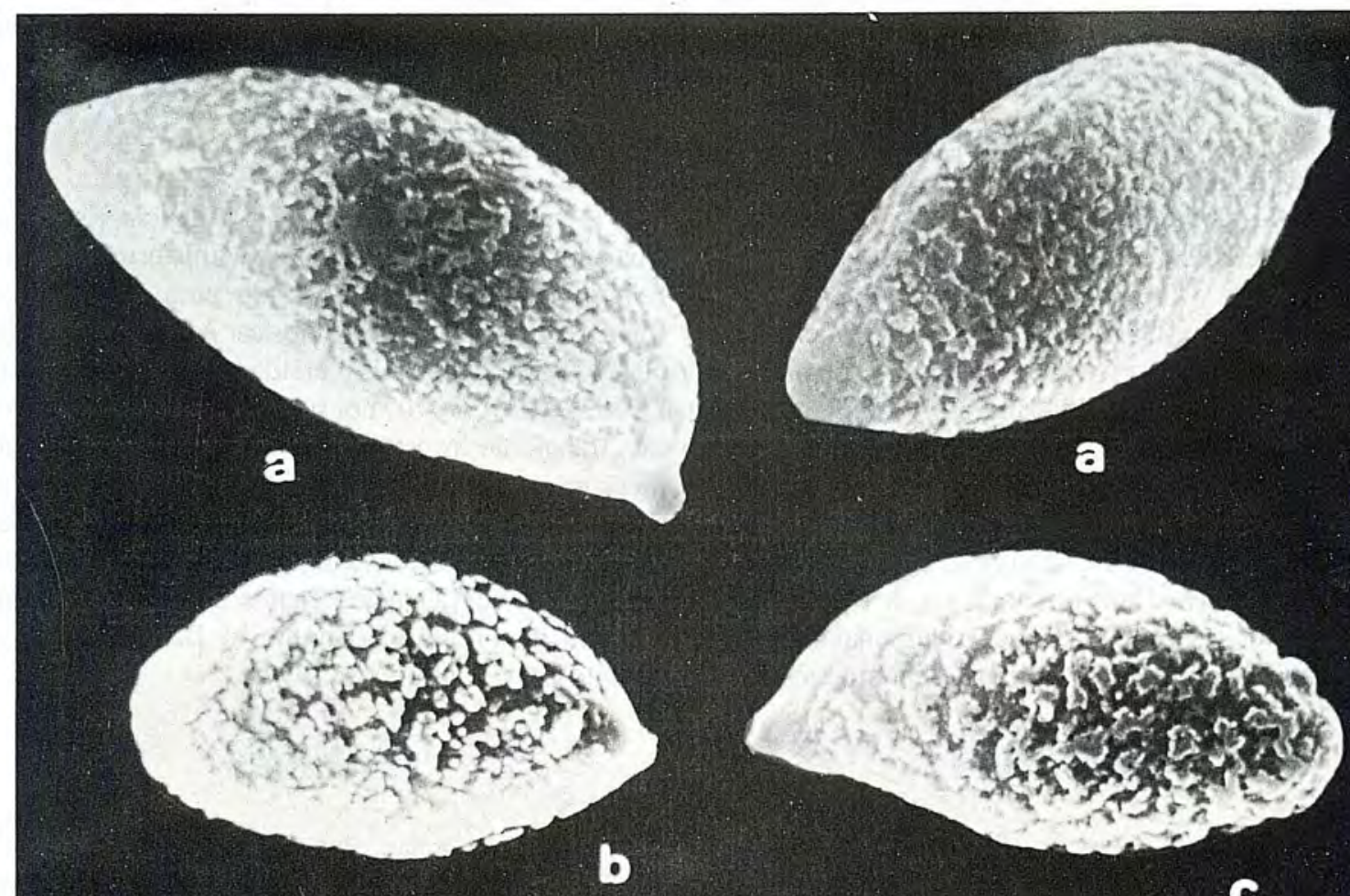

a

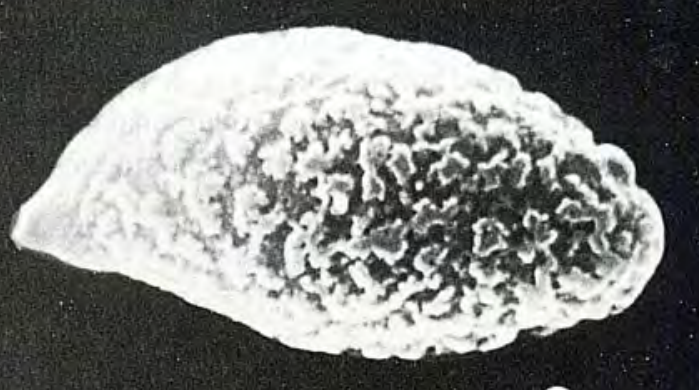

3

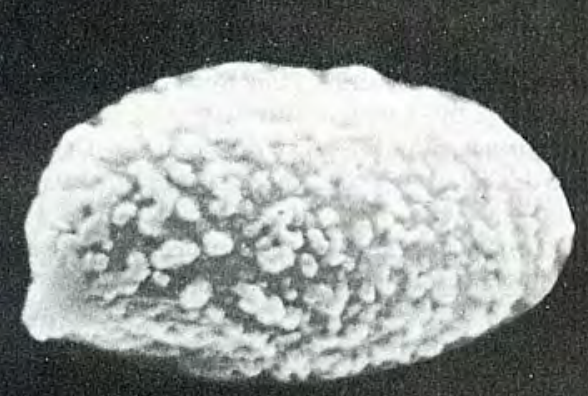

(.)

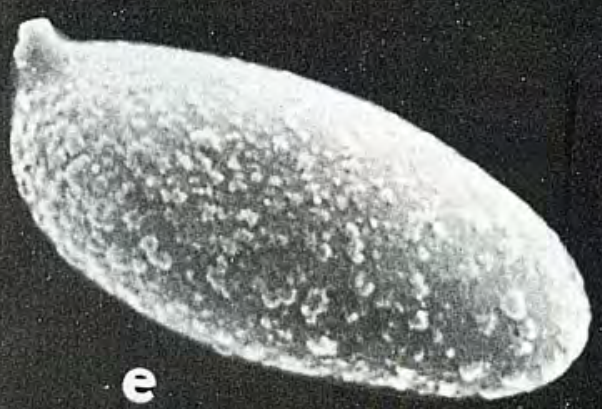

100
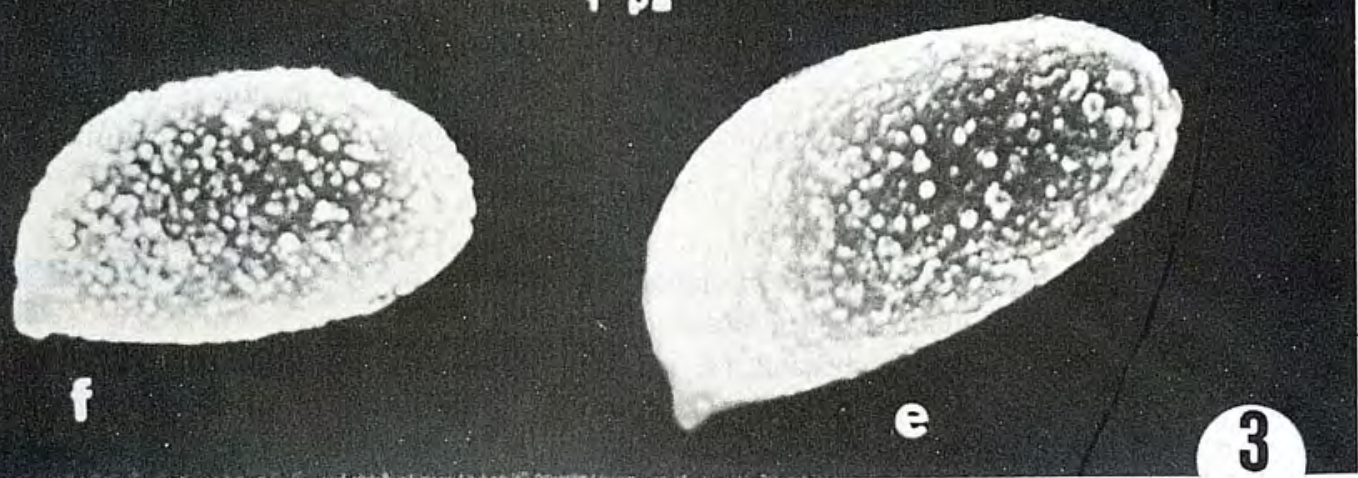

Fig. 3.- a. Cortinarius lebre (AH 15467); b, C. Luteostriatulus (AH 15465); c. C. magellanicus (AH 15458); d. C. olivaceobubalinus (AH 15466); e. C. pachynemeus (AH 15475); f. C. pugionipes (AH 15293). 
= Dermocybe oliveoicterina Moser in Moser \& Horak, Beih. Nova Hedwigia 52:492 (1975).

Cortinarius semipellucidus (Moser) G. Moreno \& Valenzuela comb. nov.

= Dermocybe semipellucida Moser in Moser \& Horak, Beilh. Nova Hedwigia 52: 497 (1975).

Cortinarius teresse (Garrido) G. Moreno \& Valenzuela. Comb. nov.

= Dermocybe teresse Garrido Biblioth. Mycol. 120:168 (1988).

Las nuevas combinaciones propuestas, se basan en los criterios señalados por Hoiland (1983), quien considera al género Dermocybe como un subgénero de Cortinarius, ya que varios de los pigmentos que se han señalado como propios para las especies de Dermocybe, han sido registra- dos para Cortinarius y por lo tanto no representan un carácter distintivo.

\section{AGRADECIMIENTOS}

Queremos agradecer a la Agencia Española de Cooperación Internacional del Ministerio de Asuntos Exteriores, Secretaría de Estado para la Cooperación Internacional y para Iberoamérica. A los proyectos S-9603 DID-UACH de Universidad Austral de Chile y FONDECYT 1970707 por su apoyo para la realización de este trabajo de investigación. Al Dr. Horak (ZT) por el préstamo de diverso material tipo de Cortinarius. Agradecemos la ayuda de J. A. Pérez, del Servicio de Microscopía Electrónica de la Universidad de Alcalá de Henares. A la Universidad de Alcalá de Henares, por la concesión de un contrato de Profesor Visitante al Dr. E: Valenzuela, durante el mes de Julio de 1997.

\section{REFERENCIAS}

Garrido, N. (1985). Index Agaricalium chilensis. Biblioth. Mycol. 99:1339

Garrido, N. (1988). Agaricaless. 1. und ilure mykorrhizen in den Nothofagus-wōldern mittelchiles. Biblioth. Mycol. 120:1-528

Hoiland, K. (1983). Cortinarius subgenus Dermocybe. Opera Bot. 71: $1-113$

Horak, E. (1980). Flora Criptogámica de Tierra del Fuego. Orden Agaricales. Tomo 11:1-524, Buenos Aires. Argentina.

Lazo, W. (1984). Introducción al estudio de los hongos superiores III. Bol. Micol. 2:27-66

Moreno, G.; Pöder,R.; Kirchmair, M.; Esteve-Raventós ,F. \&
IIcykoop,M. (1997). Dermocybe cistoadelpha, a new species in the section Sanguineae from Spain. Mycotaxon 62:239-246

Noser, M. \& Horak, E. (1975). Cortinarius Fr. und nahe verwandte gattungen in Südamerika. Beih. Nova Hedwigia 52:1-628

Valenzucla, E.; Moreno,G. \& Grinbergs, J. (1992). Agaricales sensu lato de Chile. I. Bol. Soc. Micol. Madrid 17:81-93

Valenzuela, E.; Moreno,G.; Garnica, S. \& Grinbergs, J. (1994). Agaricales sensu lato de Chile. II. Bol. Soc. Micol. Madrid. 19:281-304

Valenzuela, E. \& Esteve-Raventós, F. (1994), Cortinarius horakii, e new species from Chile. Myeol. Res. 98:937-938 